\title{
Analysis of Endocan Levels in Hypertensive Patients as Risk Factors of Chronic Kidney Disease
}

\author{
Suryani Jamal ${ }^{1}$, Uleng Bahrun ${ }^{1}$, Ibrahim Abdul Samad ${ }^{1}$, Fitriani Mangarengi ${ }^{1}$, Hasyim Kasim ${ }^{2}$, \\ Ilham Jaya Patellongi ${ }^{3}$
}

${ }^{1}$ Department of Clinical Pathology, Faculty of Medicine, Hasanuddin University/Dr. Wahidin Sudirohusodo Hospital, Makassar, Indonesia. E-mail: suryani.jamal@gmail.com

${ }^{2}$ Department of Internal Medicine, Faculty of Medicine, Hasanuddin University/Dr. Wahidin Sudirohusodo Hospital, Makassar, Indonesia ${ }^{3}$ Department of Physiology, Faculty of Medicine, Hasanuddin University/Dr. Wahidin Sudirohusodo Hospital, Makassar, Indonesia

\begin{abstract}
This study aimed to analyze endocan levels as a marker of endothelial dysfunction in the control group, patients with stage I hypertension, stage II hypertension, and patients with end-stage renal disease. Endocan levels were measured with ESM-1 (endocan) kit by Enzyme-Linked Immunosorbent Assay (ELISA) method. This study used a cross-sectional method and was conducted in Dr. Wahidin Sudirohusodo Hospital, Makassar and Hasanuddin University Hospital from September to October 2017. There were 83 samples in this study, consisting of 12 samples in the control group, 22 samples of stage I hypertension, 28 samples of stage II hypertension, and 21 samples of end-stage renal disease aged 20-90 years old. This study showed significantly higher endocan levels in patients with stage II hypertension and end-stage renal disease $(p<0.05)$. Endocan levels were significantly higher $(p<0.05)$ in patients with end-stage renal disease compared with the control group and patients with stage I hypertension; but not significantly higher $(p>0.05)$ compared to patients with stage II hypertension. Also, the median of endocan levels in patients with the end-stage renal disease was higher ( $309,850 \mathrm{ng} / \mathrm{L})$ compared to patients with stage II hypertension (273,050 $\mathrm{ng} / \mathrm{L})$.
\end{abstract}

Keywords: Endocan, endothelial dysfunction, hypertension, chronic kidney disease

\section{INTRODUCTION}

Chronic Renal Failure (CRF) is a condition characterized by a decrease in Glomerular Filtration Rate (GFR) and an increase in albumin levels in the urine. Chronic renal failure is a widespread health problem and affects $5-10 \%$ of the world's population. ${ }^{1-3}$ This disease ranks the tenth non-communicable diseases, which frequently occurs with a prevalence of $0.2 \%$ in Indonesia. ${ }^{4}$

World Health Organization (WHO) estimates that there will be an increased number of patients with end-stage CRF in Indonesia in $1995-2025$ by $41.4 \%$. Data from the Indonesian Nephrology Association (PERNEFRI) estimates that there are 70,000 people are suffering from kidney failure or end-stage CRF in Indonesia. This condition will continue to increase by around $10 \%$ each year. ${ }^{4}$

There are many markers known for impaired kidney function, despite of many shortcomings. This study is about endocan as a marker to analyze endothelial dysfunction in hypertension as a risk factor for CRF. ${ }^{5}$ Endocan, previously known as Endothelial cell-Specific Molecule 1 (ESM-1), was found to be associated with some diseases such as cancer, hypertension, kidney transplant rejection, and CRF. ${ }^{6}$ Endothelial cells in most organs secrete endocan, although the actual function of these relatively new molecules is not fully known. However, endocan play an important role in inflammation, regulation of cell adhesion molecules, endothelial cytoskeleton, and lymphocyte function. Endocan has a prognostic effect on hypertension, rejection of transplantation, and chronic renal failure.

Based on the description, the following research questions can be formulated: Are there any differences in serum endocan levels in the control group, hypertensive patients, and patients with CRF?

\section{METHODS}

The method used in this study was Sandwich-ELISA. The micro ELISA plate in this kit was coated with specific antibodies against endocan/ESM-1. ${ }^{8}$ This research was an observational cross-sectional study. This research was carried out in the Emergency Treatment Unit, Outpatients, and Internal Medicine Installation of Dr. Wahidin 
Sudirohusodo Hospital, Makassar and Hasanuddin University Hospital (RSPTN UH) for sampling and Medical Faculty of Hasanuddin University Research Unit/Hasanuddin University Hospital for specimen testing. This study was performed from September 2017 to October 2017. The minimum number of samples in this study was 18 samples for each group with 83 total samples.

All patients involved in this study had signed the informed consent and met the requirements of the Ethics Committee for Health Research (KEPK) Faculty of Medicine, University of Hasanuddin/Rumah Sakit Perguruan Tinggi Negeri Hasanuddin University (RSPTN UH)/RSUP Dr. Wahidin Sudirohusodo Makassar. This study was approved with a recommendation number of $677 / \mathrm{H} 4.8 .4 .5 .31 /$ PP36-KOMETIK/2017.

\section{RESULTS AND DISCUSSION}

Of the total 83 samples, which met the inclusion criteria, 12 patients were involved as controls. Also, 22 patients with stage I hypertension, 28 patients with stage II hypertension, and 21 patients with ESRD were found. ${ }^{9}$ The distribution of gender and age in each group can be seen in Table 1 .

From Table 1, samples can be considered homogeneous based on gender distribution. However, samples were not homogeneous based on age. Stage I hypertension was more common in patients with older age (60.5 \pm 13.4 years) compared to stage II hypertension ( $50.5 \pm 47.8$ years), ESRD ( $47.8 \pm 14.0$ years), and controls ( $30.3 \pm 12.0$ years).

Endocan levels as a risk factor for CRF in patients with hypertension was analyzed with Spearman correlation test was performed on serum endocan levels between groups (control, stage I hypertension, stage II hypertension, and ESRD); Kruskal-Wallis test followed by Mann-Whitney was used to compare the serum endocan levels in each group, because Shapiro-Wilk test showed that the data of serum endocan levels in all group except control group were not normally distributed (Table 2).

Serum endocan levels were recorded and a data distribution test was used for data transformation. Results showed that all data except the control group were normally distributed. Therefore, Oneway Analysis of Variance (ANOVA) and Least Significant Difference (LSD) were used to determine the comparative log of serum endocan levels among the groups (Table 2).

Based on the range analysis in Table 2 there was an increase of serum endocan levels in patients with stage II hypertension and ESRD proved by Spearman correlation coefficients of $r=0.298$ and $p=0.003$ $(<0.05)$. The results of the Kruskal-Wallis and Mann-Whitney test showed that serum endocan levels in patients with ESRD were significantly higher

Table 1. Characteristics of research samples

\begin{tabular}{clllll}
\hline Characteristics & $\begin{array}{c}\text { Control } \\
(\mathbf{n = 1 2})\end{array}$ & $\begin{array}{c}\text { Stage I } \\
\text { Hypertension } \\
(\mathbf{n = 2 2})\end{array}$ & $\begin{array}{c}\text { Stage II } \\
\text { Hypertension } \\
(\mathbf{n = 2 8})\end{array}$ & $\begin{array}{c}\text { ESRD } \\
(\mathbf{n = 2 1})\end{array}$ & $\mathbf{p}^{*}$ \\
\hline Gender & & & & & \\
$\quad$ Male & $5(41.7 \%)$ & $10(45.5 \%)$ & $13(46.4 \%)$ & $13(61.9 \%)$ & $0.607^{*}$ \\
$\quad$ Female & $7(58.3 \%)$ & $12(54.5 \%)$ & $15(53.6 \%)$ & $8(38.1 \%)$ & \\
Age (year) & & & & & $0.000^{* *}$ \\
$\quad$ Mean & 30.3 & 60.5 & 50.5 & 47.8 & \\
SD & 12.0 & 13.4 & 47.8 & 14.0 & \\
\hline
\end{tabular}

*Chi-Square test; **Oneway ANOVA

Table 2. Comparison of serum endocan level among the research groups

\begin{tabular}{lcccccc}
\hline \multirow{2}{*}{ Group } & \multicolumn{9}{c}{ Serum Endocan Level (ng/L) } & \multirow{2}{*}{$\mathbf{p}^{*}$} & \multirow{2}{*}{$\mathbf{p}^{* *}$} \\
\cline { 2 - 5 } & $\mathrm{n}$ & Range & Median & Mean \pm SD & & \\
\hline Control & 12 & $23.59-604,47$ & $287,115^{\text {aa }}$ & $298,193 \pm 132,756$ & 0.114 & \\
Stage I hypertension & 22 & $133,32-840,53$ & $273,050^{\text {aa }}$ & $314,648 \pm 151,512$ & 0.000 & 0.042 \\
Stage II hypertension & 26 & $194,89-689,11$ & $309,850^{\text {ab }}$ & $349,407 \pm 131,195$ & 0.001 & \\
ESRD & 21 & $208,45-1815,17$ & $393,180^{\text {bb }}$ & $528,467 \pm 383,843$ & 0.000 & \\
\hline
\end{tabular}

*Shapiro-Wilk test; **Kruskal Wallis+Mann-Whiney test; Superscript, which has the same components indicated that the Mann-Whitney test results showed $p>0.05$, whereas $p<0.05$ was significant. The result of Mann-Whitney tests showed $p>0.05$; Spearman correlation showed $r=0.298$ and $p=0.003$ between the group, with serum endocan level 
$(p<0.05)$ compared to the control group and stage I hypertension; despite insignificant difference $(p>0.05)$. The median of serum ESRD endocan levels in stage II hypertension was higher $(309,850 \mathrm{ng} / \mathrm{L})$ compared to that of stage I hypertension $(273,050 \mathrm{ng} / \mathrm{L})$.



Figure 1. Endocan level according to the subject group

* Shapiro-Wilk test; ** Kruskal-Wallis+Mann-Whitney test; Superscript, which has the same components indicated that the Mann-Whitney test results showed $p>0.05$, whereas $p<0.05$ was significant; Spearman's correlation showed $r=0.298$ and $p=0.003$ between groups with serum endocan levels

Based on the results of range analysis in Table 3, there was an increase of serum endocan levels in patients with stage II hypertension and ESRD proved by Spearman correlation with $r=0.298$ and $p=0.003$ (significant; $p<0.05$ ). The results of the Oneway ANOVA and LSD test showed that serum endocan levels in patients with ESRD were significantly higher $(p<0.05)$ compared to the control group and stage I hypertension; despite an insignificant difference $(p>0.05)$. The mean \pm SD log ESRD of serum endocan levels in the ESRD group was higher compared to stage II hypertension $(2.644 \pm 0.352$ vs. $2.519 \pm 0.144 \mathrm{ng} / \mathrm{L})$.



Figure 2. Log k endocan level based subject groups

From Figure 2, it was shown that although there was a higher tendency of increase of log mean serum endocan levels towards ESRD, but only the ESRD group had a greater difference of mean value. Mean in the ESRD group seemed to differ from the mean in the other three groups (control, stage I hypertension, and stage II hypertension) and the variation of log data of serum endocan levels in ESRD was dominant to the higher direction.

A study of the endocan levels was performed in Dr. Wahidin Sudirohusodo Hospital, Makassar and RSPTN/Hasanuddin University Makassar during September-October 2017. There were 83 samples, which met the sample criteria with 12 patients as controls, 22 patients with stage I hypertension, 28 patients with stage II hypertension, and 21 patients with ESRD. The four groups were considered homogeneous based on the distribution of gender, but not based on age. There were a greater number of patients of older age in the group with stage I hypertension (60.5 \pm 13.4 years) compared to stage II hypertension ( $50.5 \pm 47.8$ years), ESRD ( $47.8 \pm 14.0$ years), and controls ( $30.3 \pm 12.0$ years). It indicated that age was able to make bias in differences in levels of serum endocan levels among groups (Table 1 ).

There was a pattern of increase of serum endocan levels in patients with stage II hypertension and

Table 3. Comparison of the log endocan level to determine the data distribution

\begin{tabular}{|c|c|c|c|c|c|c|}
\hline \multirow{2}{*}{ Group } & \multicolumn{4}{|c|}{ Log Endocan Level (ng/L) } & \multirow{2}{*}{$\mathbf{p}^{*}$} & \multirow{2}{*}{$\mathbf{p}^{* *}$} \\
\hline & $n$ & Min/Max & Median & Mean \pm SD & & \\
\hline Control & 12 & $1.37 / 2.78$ & 2.458 & $2.399 \pm 0.344^{\mathrm{aa}}$ & 0.000 & \\
\hline Stage I hypertension & 22 & $2.12 / 2.92$ & 2.436 & $2.463 \pm 0.168^{\mathrm{aa}}$ & 0.109 & 0.011 \\
\hline Stage II hypertension & 26 & $2.29 / 2.84$ & 2.491 & $2.519 \pm 0.144^{\mathrm{ab}}$ & 0.095 & \\
\hline ESRD & 21 & $2.32 / 3.26$ & 2.595 & $2.644 \pm 0.352^{\mathrm{bb}}$ & 0.154 & \\
\hline
\end{tabular}

*Shapiro-Wilk test; **Oneway ANOVA+LSD test; superscript has the same component, LSD test results showed $p>0.05$. $p<0.05$ was significant. Spearman correlation $(r=0.298$ and $p=0.003)$ between the group with endocan serum level 
ESRD. The results of the Kruskal-Wallis and Mann-Whitney tests showed that endocan levels in patients with ESRD were significantly higher than those in the control group and stage I hypertension, although not significantly different from stage II hypertension. However, the median of serum endocan level in patients with ESRD group was higher compared to patients with stage II hypertension (Table 2). Although there is a higher tendency of the median of serum endocan levels to the direction of ESRD, only the ESRD group had a relatively different median value. There was a greater difference in the median of the ESRD group compared to the other three groups (control, stage I hypertension, stage II hypertension (Figure 1).

The pattern indicated by the log of serum endocan level increased when the subjects had stage II hypertension and ESRD. The results of the Oneway ANOVA and LSD test showed that serum endocan level in patients with ESRD was significantly higher compared to the control group and stage I hypertension; although not significantly different from stage II hypertension group, the mean \pm SD log of ESRD endocan level was higher than stage II hypertension (Table 3). This was in accordance with a study by Afsar, suggesting that higher endocan levels were found in advanced CRF and endocan levels were correlated with GFR. ${ }^{\text {? }}$

According to Figure 2, there was a higher tendency of mean log endocan levels towards ESRD, but only the ESRD group with a slightly greater difference of mean value. Mean in ESRD group seemed to differ from that of the other three groups (control, stage I hypertension, and stage II hypertension) and the variation of log data of endocan level ESRD was dominant in the higher direction. This was consistent with a study by Afsar, suggesting that there was a significant decrease of kidney function in the group with higher serum endocan levels.

The following is a study of the relationship between plasma endocan levels and inflammation, endothelial dysfunction, cardiovascular events, and survival of chronic kidney failure. Plasma endocan concentrations are negatively correlated with estimated glomerular filtration rates but positively correlated with inflammatory markers such as PTX3 and hsCRP. Also, endocan levels are associated with endothelial dysfunction evaluated by Flow-Mediated Vasodilatation (FMD) and Carotid Intima-Media Thickness (CIMT). After adjusted for multiple confounders, endocan levels are independently associated with cardiovascular events. ${ }^{10-15}$
A study by Yilmaz et al. showed that serum endocan levels based on estimated Glomerular Filtration Rate (eGFR) in patients with stage I, stage II, stage III, and stage IV chronic renal failure was $1.2 \mathrm{ng} / \mathrm{mL}$, $2.8 \mathrm{ng} / \mathrm{mL}, 6.6 \mathrm{ng} / \mathrm{mL}$, and $13.3 \mathrm{ng} / \mathrm{mL}$, respectively. This pattern is similar to the hs-CRP protein because of the inflammatory process. ${ }^{16}$ Nevertheless, the reason why serum endocan levels are significantly higher in chronic kidney failure than other diseases remains to be elucidated. An increase in serum endocan levels in CRF likely results from decreased clearance. Therefore, it is possible to carry out the evaluation together with urine endocan levels. ${ }^{17-19}$

The medical history and physical test in this study were one of the limitations in this study; therefore, the possibility of the factors, which might affect the endocan levels as lipid profile that expresses endocan was unable to be identified.

\section{CONCLUSION AND SUGGESTION}

Endocan levels increased in patients with stage II hypertension and ESRD. Endocan levels in patients with ESRD were significantly higher than those in the control group and stage I hypertension; although not significantly different from the stage II hypertension group. Also, the median of serum endocan levels in patients with ESRD was higher compared to stage II hypertension. Therefore, further research was needed to determine the relationship between endocan levels and the stage of chronic kidney failure. Studies about urine endocan levels are needed to determine whether increased serum endocan levels in patients with CRF are the result of increased secretion or decreased renal clearance. Further research is needed to compare endocan levels in serum with other markers such as markers of endothelial dysfunction.

\section{REFERENCES}

1. JNC VII. The seventh report of the Joint National Committee on prevention, detection, evaluation, and treatment of high blood pressure. JAMA, 2003; 289: 2560-71.

2. CDC. National chronic kidney disease fact sheet: General information and national estimates on chronic kidney disease in the United States. Atlanta: US Department of Health and Human Services. Centers for Disease Control and Prevention. 2014. Available at: http://www.cdc.gov/diabetes/pubs/factsheets/kidne y.htm (accessed 15 February, 2017)

3. KDIGO. Clinical practice guideline for the evaluation and management of chronic kidney disease. Kidney International Supplements, 2013; 3(1): 63-73. 
4. Basic Health Research, Health Research and Development Agency Ministry of Health Republic of Indonesia. 2013; 95-103.

5. Kali A, Shetty KSR. Endocan: A novel circulating proteoglycan. Indian J Pharmacol, 2012; 46 (6): 579-583.

6. Kang YH, Ji NY, Han SR, Lee CI, Kim JW, et al. ESM-1 regulates cell growth and metastatic process through activation of NF- k B in colorectal cancer. Cell Signal, 2012; 24(10): 1940-9.

7. Afsar B, Takir M, Kostek O, Covic A, Kanbay M. Endocan: A new molecule playing a role in the development of hypertension and chronic kidney disease. The Journal of Clinical Hypertension, 2014; 16 (12): 914-916.

8. Human ESM-1 (Endothelial Cell Specific Molecule 1) ELISA Kit. Available at: http://www.elabscience.com (accessed 2 April, 2017).

9. Bakri S, Lawrence GS. Genetics of hypertension. In: Lubis HR. Hypertension and kidney: In the framework of full service Prof. Dr. Harun Rasyid Lubis, Sp.PD-KGH. Field. USU Press, 2008; 19-31.

10. Balta S, Mikhailidis DP, Demirkol S, Ozturk C,


indicator in newly diagnosed patients with hypertension: A pilot study. Angiology, 2013; 1-5.

11. Campbell D, Weir MR. Defining, treating, and understanding chronic kidney disease-a complex disorder. The Journal of Clinical Hypertension, 2015; 17(7): 514-27.
12. Chen J, Hamm LL, Mohler ER, Hudaihed A, Arora R, et al. Interrelationship of multiple endothelial dysfunction biomarkers with chronic kidney disease. PLos One, 2015; 10(7): 1-10.

13. Glancy MR. Endocan as a potential diagnostic or prognostic biomarker for chronic kidney disease. Kidney International, 2014; 86: 1079-81.

14. Kose M, Emet S, Akpinar TS, Kocaaga M, Cakmak R, et al. Serum endocan level and the severity of coronary artery disease: A pilot study. Angiology, 2015; 66(8):727-31.

15. Lee HG, Choi HY, Bae JS. Endocan as a potential diagnostic or prognostic biomarker for chronic kidney disease. Kidney International, 2014; 86: 1079-81.

16. Yilmaz MI, Siriopol D, Saglam M, Kurt YG, Unal HU, et al. Plasma endocan levels associate with inflammation, vascular abnormalities, cardiovascular events, and survival in chronic kidney disease. Kidney Int, 2014; 86: 1213-20.

17. Rahmania L, Cortes DO, Irazabal M, Mendoza $M$, Santacruz $C$, et al. Elevated endocan levels are associated with development of renal failure in ARDS patients. Intensive Care Med Exp, 2015; 3(1): A264.

18. Su YH, Shu KH, Hu CP, Cheng CH, Wu MJ, et al. Serum endocan correlated with stage of chronic kidney disease and deterioration in renal transplant recipients. Transplant Pro, 2014; 46(2): 323-7.

19. Whayne TF. Endocan in hypertension and cardiovascular disease. Angiology, 2016; 20: 1-3. 